Research Article

\title{
Energy-Efficient Coalition Games with Incentives in Machine-to-Machine Communications
}

\author{
Raymond W. Juma (D), Anish M. Kurien, and Thomas O. Olwal \\ Department of Electrical and Electronic Engineering, Tshwane University of Technology, Pretoria, South Africa \\ Correspondence should be addressed to Raymond W. Juma; rjwekesa@gmail.com
}

Received 14 February 2019; Revised 13 May 2019; Accepted 23 May 2019; Published 16 June 2019

Guest Editor: Huan Zhou

Copyright ( $\odot 2019$ Raymond W. Juma et al. This is an open access article distributed under the Creative Commons Attribution License, which permits unrestricted use, distribution, and reproduction in any medium, provided the original work is properly cited.

\begin{abstract}
The need to achieve energy efficiency in machine-to-machine (M2M) communications has been a driver of the use of coalition game-based cooperative communication schemes. The proposed schemes have shown good energy-efficient performance results in the recent past. However, sustaining cooperation amongst coalition games of M2M devices from different network-operating authorities requires appropriate incentives. A review of the literature demonstrates that a limited number of contributions have considered the use of coalition games with incentives in $\mathrm{M} 2 \mathrm{M}$ communications. In this paper, an energy-efficient coalition game with incentives in M2M communications is proposed. This work considers a Sierpinski triangle technique to partition M2M devices into multiple networks of hierarchical zones. Based on the constructed zones, a contract-modelled incentive is invoked to stimulate multihop transmissions between devices up to the BS/sink. The results obtained demonstrate that the proposed approach is on average $10 \%$ more energy efficient than the closely related existing algorithm, the coalition game theoretical clustering (CGTC).
\end{abstract}

\section{Introduction}

The introduction of M2M communications has catapulted the automation of various tasks in real time. The applications of M2M have been embraced today in the sectors of transport, military, healthcare, and smart cities. However, despite the growing interest of M2M technologies, one of the greatest challenges faced by $\mathrm{M} 2 \mathrm{M}$ devices is the limited battery lifetime of operations. There is thus a need to prolong the operation of M2M devices while ensuring a stable quality of service/quality of experience (QoS/QoE). This can be achieved through cooperation among the devices in the network. Zhou et al. [1] utilise the data offloading technique through vehicular ad hoc networks (VANETs). Huang et al. [2] describe the use of credit-based clustering (CBC) scheme to encourage sharing among devices in the same social network. The application of cooperative schemes in energyefficient management has been proposed by Raymond et al. [3] and Olwal et al. [4]. Cooperative schemes that invoke game theory are examined for energy efficiency in WSNs [5].
Over years, the application of game theory has had a considerable impact on a sizeable number of disciplines that include engineering, economics, political science, philosophy, and even psychology [6].

In recent years, game theory has been applied in the analysis of communication networks. In an effort to minimise energy consumption in a network, an efficient-energy consumption protocol (EECP) utilises fixed clusters and applies the random weight technique in selection of cluster heads ( $\mathrm{CHs})$ [7]. The concept of game theory is applied during multihop data transmission to the sink. A cooperative game algorithm for routing purposes which considers rewarding cooperative devices and punishing noncooperative devices is proposed for energy management in WSNs [8].

A coalition game takes into consideration the benefits of all the players in the network; players adopt strategies that strengthen the utility of another player. It may be considered an appropriate approach when considering its application in the implementation of fair and well- 
organised cooperative strategies in communication networks [9]. Through games of coalitions, it has been argued by Bacci et al. [10] that huge computation overheads that are associated with larger networks may be avoided. AlSkaif et al. [11] argue that coalitional games are characterised by group formation that demonstrates better performance in energy efficiency when compared to noncooperative games A further benefit of coalition game theory (CGT) is demonstrated through the reduction of power consumption in a WSN that is achieved as a result of the formation of coalition structures [12]. Under these structures, players choose strategies to maximise their own utility as a group. Such group-based strategies enable individuals to consume less energy and operate for a longer period than when each player could have strategized independently. Earlier studies had explained that a selfish node or device in a communication network caused network performance degradation $[13,14]$. Due to the distance effect, the devices that were located farther from each other would consume more energy before reaching the processing device/sink.

The need to achieve energy efficiency in M2M communications has been attained through the use of coalition game-based cooperative communication schemes $[5,7,15]$. However, sustaining cooperation amongst coalition games of M2M devices from different network-operating authorities or regions is a great challenge. This is due to the selfish behaviour of some of the devices or operating authorities that prefer to conserve their energy instead of consuming it to assist other devices to reach the sink/BS. To mitigate this challenge, the study proposes a coalition game theoretical clustering with incentive (CGTCI) algorithm. The novel approach proposed considers a Sierpinski triangle technique to partition distributed M2M devices into multiple networks of hierarchical zones. Based on the constructed zones, coalition structures supervised by the devices elected as cluster heads are developed in the zones located far from the BS. Devices closer to the BS are not organised into coalition structures. A contract model-based incentive is invoked to stimulate multihop transmissions up to the BS/sink. The main contributions of this work can be summarised as follows:

(i) A novel algorithm, coalition game theoretical clustering with incentive (CGTCI), is proposed to minimise energy consumption in M2M communications.

(ii) The proposed algorithm CGTCI is designed which starts with the partitioning of M2M devices into multiple networks of hierarchical zones, formation of coalition structures in the created hierarchical multiple zones, and invoking of a contract-modelled incentive to stimulate multihop transmissions up to the $\mathrm{BS} /$ sink.

(iii) It is demonstrated through simulations that the proposed algorithm, CGTCI, improves energy efficiency among the M2M communications when compared with the closely related traditional approaches: CGTC [15], CG-DC (an improvement of low-energy adaptive clustering hierarchy $(\mathrm{LEACH})$ )
[16], Raymond et al. [3], and noncoalition game (NCG) algorithms.

The remainder of this paper is organised as follows: Section 2 presents related works on coalition games. Section 3 introduces the fundamentals of the proposed algorithm. In Section 4 , the system model is described. Section 5 describes the proposed solution. In Section 6, the performance and simulation results are evaluated. Section 7 finally highlights conclusions and future work.

\section{Related Works}

The discussions of coalition games have been extensively presented in the existing literature. This section leverages such contributions made previously to review the most related and credible studies regarding energy efficiency in communication networks. The hybrid game theory and a distributed cluster technique are applied in WSNs to control energy consumption by Yang et al. [17]. Each node has a payoff that is designed based on various parameters that include node degree and distance to the base station. Based on this approach, each node computes its equilibrium probability by applying the parameters of the game. The application of equilibrium probability by the node enables it to decide its suitability of being a cluster head $(\mathrm{CH})$. The node attains a good trade-off between minimizing energy dissipation and providing the required services effectively.

Yue et al. [18] propose a coalition game model which has been derived by integrating both the Markov process and theoretical approach for energy-efficient WSNs. The performance tests of the proposed model show that the lifetime and effective reachability for low-density WSNs are increased. However, the model was not evaluated for a dense network to ascertain its effectiveness of energy efficiency.

$\mathrm{Wu}$ et al. [19] examine data transfer strategies that are specified in relation to the proportion of the data sent by a node and that of the data forwarded by a node for energyefficient WSNs. The formation of coalitions is based on a Markov process. The concept of determining the absorption coefficient to measure the coalitional profiles is introduced. Nash equilibrium (NE) is used to determine the formed coalitions' approximate data transfer strategies. However, finding the exact $\mathrm{NE}$ in this proposal is a nondeterministic polynomial (NP) time complex problem. It is advised that a low computationally complex alternative strategy be considered.

Jing and Aida [20] present a cooperative game theoretical model for clustering algorithms in which nodes balance the energy consumption and maximise their network lifetime. The selfish behaviour of nodes in noncooperative games expedites network partition and results in an unfair residual energy distribution within the network. The work by Jing and Aida [20] considers an algorithm in which there is a trade-off in the individual cost and the network-wide cost of sensor nodes in forming a coalition. In this respect, a candidate cluster head $(\mathrm{CCH})$ cooperates with a node considered to be close and almost equivalent in terms of energy (node with redundant energy), and the whose 
transmission covers a long distance. The Shapley value that has anonymity, dummy property, and additivity property is introduced to assign a single cost allocation to the costsharing game. The Shapley value through random ordering of nodes provides a relatively anonymous solution, where agents' change of names does not alter their cost shares. However, the initial candidate selection in this approach is partly random and does not guarantee the choice of the most suitable candidate as a $\mathrm{CH}$. In this approach, the direct communication between the selected device and the BS contradicts the scalability benefits achieved with the coalition game clustering.

Afsar [15] shows how a coalitional game theoretical clustering (CGTC) algorithm can be used to control energy consumption in WSNs through the adoption of multihop communications. The coalitions are demarcated into two major groups based on the location (i.e., either far or in the vicinity) of sensor nodes in relation to the BS. A set of nodes with the highest residual energy in the far region are referred to as coalition head nominees (CHNs) which initiate cooperative games within their surroundings. The CHNs along with two other nodes then shape final coalitions. The vicinity region on the contrary considers that small coalitions are formed to tackle the energy-consuming data-relaying task. In the CGTC approach, local parameters such as residual energy, number of neighbours, and proximity to the BS are important in forming the coalitions since the main objective is energy efficiency (EE). The Shapley value is applied to distribute the average of marginal contributions to coalitions generated. However, the random candidate election could easily result in the acceleration to a dead state in cases where a candidate of low residual energy is picked as a cluster head.

Miao and $\mathrm{Xu}$ [21] discuss a power control solution that is based on the trade-off between energy efficiency and end-toend delay that is applied as a technique to improve energy consumption in WSNs. A cooperative coalitional game is proposed to obtain a power control solution that achieves a fair distribution of the total cost amongst sources. It was observed that the minimization of delay is achieved by minimizing the remaining energy level. Each source node seeks to minimise its utility function of the discounted sum of transmission power increased cost and the source-to-sink delay cost. The Shapley value is used as a solution of the cooperative allocation game for fairness dissemination of the gains achieved as evaluated by Yeung and Petrosjan [22].

Tan et al. [23] develop a bidirectional cooperative clustering model that applies a cost-sharing game for EE. The algorithm examines the cooperation of cluster members and cluster heads ( $\mathrm{CHs}$ ) for reducing energy consumption in the network. The authors highlight that an algorithm based on game theory can be applied in the $\mathrm{CH}$ selection for the purpose of energy efficiency [24]. The algorithm is based on the subgame perfect Nash equilibrium (SPNE) approach which is used to find the Nash equilibrium (NE) in every subgame of the real game. The selection of the $\mathrm{CHs}$ relies on the SPNE technique.

Romero et al. [25] examine a game theory-based strategy to reduce energy consumption in cognitive WSNs. The strategy was initially meant to provide an answer to the problem of spectrum inefficiency. The proposed technique was shown to improve energy consumption through its ability to switch communication channels. The approach makes use of a decision strategy of changing the transmission channel depending on the behaviour of the rest of the network nodes through the application of a game theoretic technique.

As presented above, most of the related works are contextualised to traditional WSN-based M2M communications. In modern times, neither the devices in a network that constitutes M2M communications are homogeneously WSNs nor do the composing nodes share the same network operator. This makes the approach for the management of cooperative communications in M2M networks to be different from how WSNs are managed. The current sequel proposes a new solution that is a modification of the coalition game theoretical clustering (CGTC) proposed by Afsar [15]. The proposed approach considers the application of coalition game theoretical clustering that invokes an incentive scheme (CGTCI). The proposed approach aims at stimulating cooperation amongst the M2M devices that are not necessarily from the same operator. The incentive paves the way for transmissions at short distances in the hierarchical partitioned network which has an overall effect of reduced energy consumption in the network.

\section{Fundamentals of the Proposed Algorithm}

This section presents the basics of the coalition game and radio model as fundamentals of the proposed algorithm.

3.1. Basics of Coalition Game. A coalitional game can be an ordered pair $(N, V)$, in which $N$ is a finite set of players referred to as the grand coalition and the characteristic function $V$ is described as $V: 2^{N} \longrightarrow R$. In the coalition formation game, coalition structures which are partitions of the grand coalition $N$ are constructed and defined as $S=\left\{S_{1}, \ldots, S_{i}, \ldots, S_{K}\right\}$. In our future discussions, the following properties of coalition games will be considered.

3.1.1. Individual Rationality. A player $n$ will be a member of a coalition, only when the gains achieved by being in the coalition are more than those when acting selfishly or being a free rider. This can be expressed as $X_{n}>V(\{n\})$, where $X_{n}$ is the utility factor of a player in a coalition and $V(\{n\})$ is the utility factor of a free rider.

3.1.2. Group Rationality. The sum of the payoffs of a coalition should be at least the value of the coalition. This is represented as $\forall C \subseteq N$ and $X(C) \geq V(C)$. Here, $\forall C$ represents all the coalitions, $N$ is the grand coalition, $V(C)$ is the value of the coalition, and $X(C)$ is the payoff to the coalition.

3.2. Radio Model. The computation of the energy consumed during data transmission from sources to the sink considers the radio model that is illustrated in Figure 1 [26]. The 


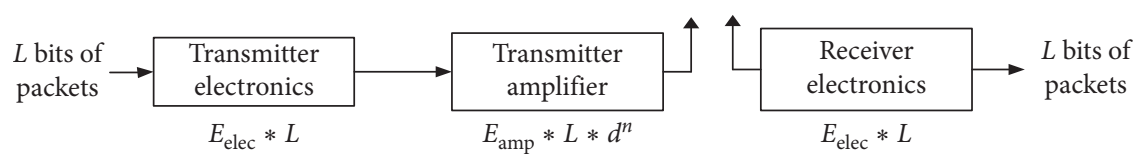

Figure 1: Radio model for energy dissipation.

energy for data transmission is proportional to distance $(d)$ and the amount of data bits $(L)$.

Accordingly, the energy needed to transmit $L$ bits of data packets through distance $d$ is expressed as

$$
\begin{aligned}
& E_{\mathrm{TX}}(L, d)=E_{\mathrm{TX}(\mathrm{elec})}(L)+E_{\mathrm{TX}(\mathrm{amp})}(L, d), \\
& E_{\mathrm{TX}}(L, d)= \begin{cases}L\left(E_{\mathrm{elec}}+\epsilon_{\mathrm{fs}} d^{2}\right), & d \leq d_{o}, \\
L\left(E_{\mathrm{elec}}+\epsilon_{\mathrm{mp}} d^{4}\right), & d>d_{o} .\end{cases}
\end{aligned}
$$

The energy expended in receiving the data packets is expressed as

$$
E_{\mathrm{RX}}=E_{\mathrm{RX}(\text { elec })}(L) .
$$

In equations (1) and (2), $E_{\text {elec }}(L)$ is the energy consumption required to run the transmitter circuitry for an $L$ bit packet; $E_{\mathrm{TX}(\mathrm{amp})}(L, d)$ is the energy consumed when an amplifier transmits $L$ bits through a distance $(d) ; \epsilon_{\mathrm{fs}}$ and $\epsilon_{\mathrm{mp}}$ are the amplification energy of free space and multipath models, respectively; and the threshold $d_{o}$ is expressed as $d_{o}=\sqrt{\left(\epsilon_{\mathrm{fs}} / \epsilon_{\mathrm{mp}}\right)}$.

This section has presented fundamentals that will be considered during the discussion of the proposed algorithm. In the next section, the modelling of the proposed algorithm is highlighted.

\section{System Model}

This section presents a detailed description of the proposed system model. Consider a single cell with one BS and a set of $N$ heterogeneous $\mathrm{M} 2 \mathrm{M}$ devices $N=\{1,2, \ldots n\}$ that are randomly distributed within the coverage area of the BS. To balance the load in the network, the network area is partitioned into hierarchical zones. Figure 2 shows the structural representation of the proposed system model.

Devices in the outer zones are organised into coalition structures. Each coalition structure is supervised by a $\mathrm{CH}$. The $\mathrm{CH}$ receives, aggregates data packets of devices associated with it, and then forwards them to selected $\mathrm{CHs} /$ devices in the hierarchical zones. The devices in the zone closer to the BS act as gateways, and they do not join coalition structures. They receive and aggregate data packets from $\mathrm{CHs}$ of the outer zones and supplement them with their own before forwarding to the BS [27]. In this network, two links are defined: backhaul and access links. The latter links the inner zone devices to the BS, while the former links devices in the coalition structure to the $\mathrm{CH}$.

The details of the proposed approach are presented in the following forms: network partitioning, coalition formation, and contract model-based incentive.
4.1. Creation of Network Partitions. The partitioning of the network considers the application of the Sierpinski triangle technique [28]. To achieve network partitioning, the following steps are followed:

(i) Consider the area of a single cell with BS as an equilateral triangle

(ii) Divide the triangle into four smaller congruent equilateral triangles

(iii) Repeat step (ii) with each of the remaining smaller triangles

(iv) For creation of three partitions, introduce curved lines to join bases of triangles that have contact with the major triangle

(v) Triangles in each partition represent the maximum number of subcluster heads that can be selected by the cluster head

Steps (i)-(v) described above are illustrated in Figure 3.

4.2. Formation of Coalition Structures. The formation of coalition structures starts with the generation of cluster heads in the respective zones. The devices choose the optimum cluster based on the calculated utility function.

4.3. Election of Cluster Heads (CHs). In the selection of the cluster head $(\mathrm{CH})$, a device with the highest cost function is elected as a $\mathrm{CH}$ in each zone [29]. The elected $\mathrm{CH}$ plays the role of selecting sub-CHs in the zone whose cost function is above the average threshold. The parameters that determine the cost function of a device are ratio of residual to initial energy $\left(E_{\mathrm{r}} / E_{\text {init }}\right)$, trust level $\left(T_{n}\right)$, node degree $(Q)$, and device data transmission energy to the $\mathrm{BS}\left(E_{\mathrm{TX}}(L, \mathrm{BS})\right)$. Their corresponding weights are given as $\alpha_{1}, \alpha_{2}, \alpha_{3}$, and $\alpha_{4}$, respectively. The cost function is defined as

$$
\operatorname{cost}(n)=\left[\alpha_{1}\left(\frac{E_{\mathrm{r}}(t)}{E_{\text {init }}}\right)+\alpha_{2} T_{n}+\alpha_{3} Q+\alpha_{4} E_{\mathrm{TX}}(L, \mathrm{BS})\right](t),
$$

where $E_{\text {init }}$ is the initial device energy at time $t=0, E_{\mathrm{r}}(t)$ stands for the current device residual energy, and $T_{n}$ is the device trust level. The trust level describes certainty of the relay nodes based on the success of achieving the objective of delivering data packets to the sink. $E_{\mathrm{TX}}(L, \mathrm{BS})$ denotes the device transmission energy used for packet transmission to the BS. The sum of the cost weights $\alpha_{1}, \alpha_{2}, \alpha_{3}$, and $\alpha_{4}$ equals unity.

4.4. Coalition Structures. The set of players in a coalition game is represented as $N=\{1,2, \ldots, n\}$; devices elected as 


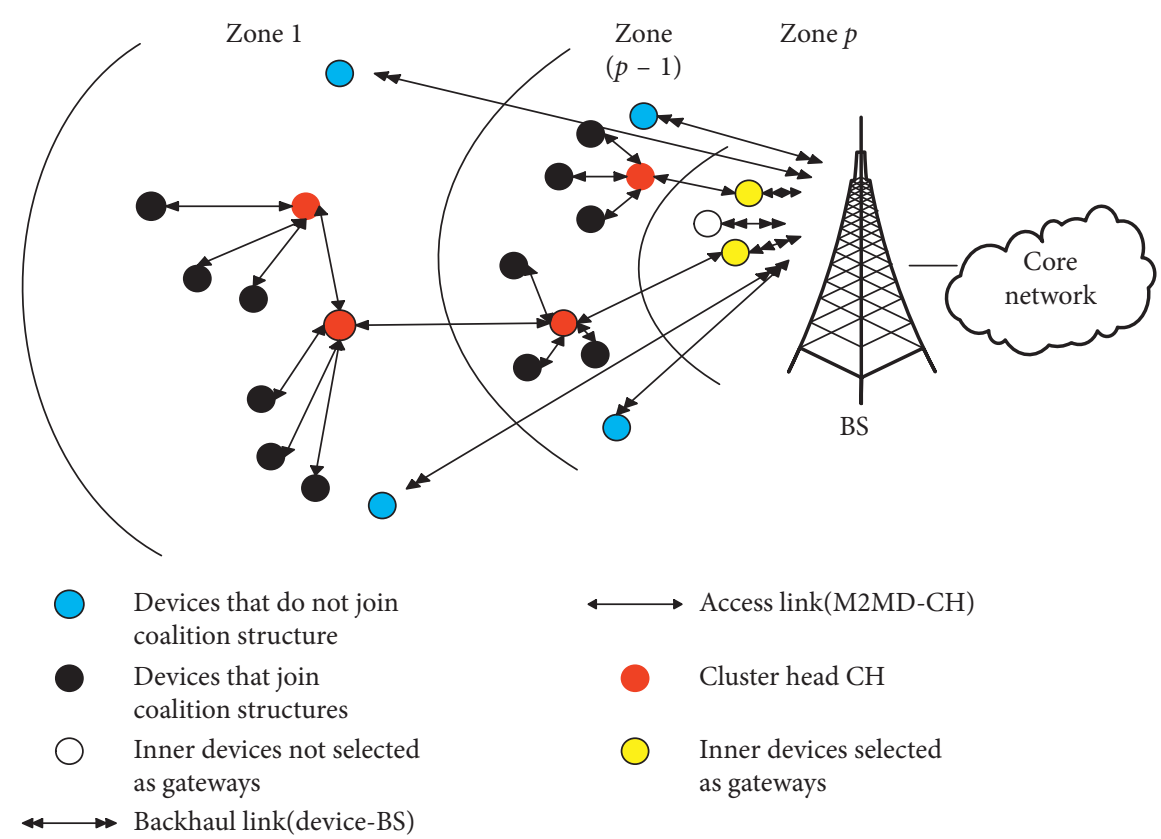

FIGURE 2: Structural representation of the proposed system model.

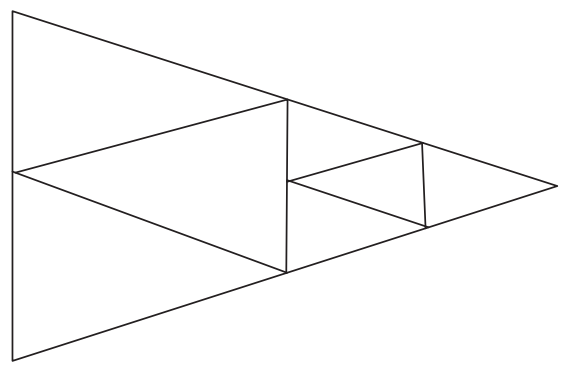

(1) Triangle divided into four congruent triangles

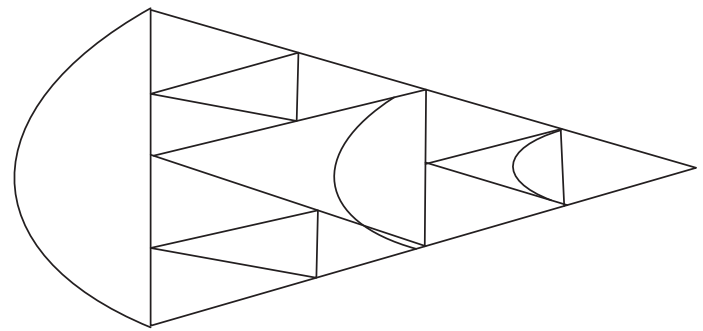

(2) Smaller remaining triangles are divided and using curved lines joined bases of triangles that have contacts with the major triangle

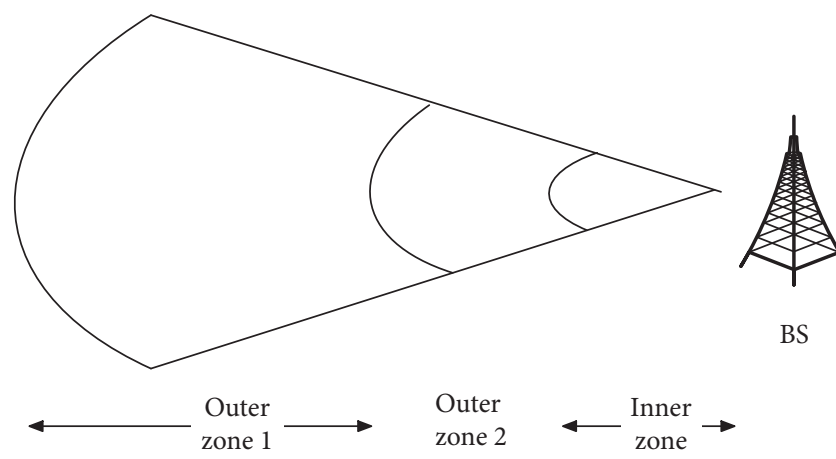

(3) Complete partitioning of a single cell with BS

FIgURE 3: Creation of network partitions.

cluster heads in the zones are defined as $\mathrm{CH}_{A(i)}$ and $\mathrm{CH}_{B(i)}$, $i=\{1,2, \ldots, M\}$, for zones A and B, respectively. The players in these zones have an option of joining a coalition or not. In either way, it depends on the computed utility factor $\left(U_{\mathrm{f}}\right)$. A device will opt for high $U_{f}$, as this signifies less energy consumption. During the computation of $U_{\mathrm{f}}$, energy used and interference encountered as data packets transmitted from the source to the sink are considered. The expression of $U_{\mathrm{f}}$ is given as [30]

$$
U_{\mathrm{f}}\left(E_{\mathrm{TX}}(L, d), \theta_{\mathrm{sn}}\right)=\left(\frac{R_{\mathrm{t}}}{E_{\mathrm{TX}}(L, d)}\right)\left(1-e^{-0.5 \mathrm{sn}}\right)^{L},
$$

where $U_{\mathrm{f}}$ is the utility factor of node $n$; $E_{\mathrm{TX}}$ is the energy required to transmit data packets, as given in equation (1), 
and depends on distance $(d)$ and data packets $(L) ; \theta_{\text {sn }}$ is the signal-to-interference-and-noise ratio (SINR) experienced by node $n$ during data transmission; and $R_{\mathrm{t}}$ is the rate at which information is transmitted to the $\mathrm{BS} / \mathrm{sink}$. The main objective for each node is to maximise its utility factor that is determined by the distance $(d)$, data packets $(L)$, and SINR $\left(\theta_{\mathrm{sn}}\right)$. The utility factor for a node joining a coalition structure $\left(U_{\mathrm{fc}}\right)$ having $L$ packets, $d_{\mathrm{c}}$ distance, and $\theta_{\text {snc }}$ interference is given as [30]

$$
U_{\mathrm{fc}}\left(E_{\mathrm{TX}}\left(L, d_{\mathrm{c}}\right), \theta_{\mathrm{snc}}\right)=\left(\frac{R_{\mathrm{t}}}{E_{\mathrm{TX}}\left(L, d_{\mathrm{c}}\right)}\right)\left(1-e^{-0.5 \theta_{\mathrm{snc}}}\right)^{L} .
$$

The utility factor for a node that considers free riding has distance and interference denoted as $d_{\text {fr }}$ and $\theta_{\text {snfr }}$, respectively, and packets denoted as $L$. The utility factor of a free riding node can be expressed as

$$
U_{\mathrm{fr}}\left(E_{\mathrm{TX}}\left(L, d_{\mathrm{fr}}\right), \theta_{\mathrm{snfr}}\right)=\left(\frac{R_{\mathrm{t}}}{E_{\mathrm{TX}}\left(L, d_{\mathrm{fr}}\right)}\right)\left(1-e^{-0.5 \theta_{\text {snfr }}}\right)^{L} .
$$

Different parameters in each case result in two values of utility factors being obtained. The utility factor of joining a coalition and that of free riding are compared. The option that generates a higher utility factor is selected by the device as it signifies less transmission energy and therefore energy saving.

For the nodes in the coalition structure, the respective $\mathrm{CHs}$ through the time-division multiple access (TDMA) establish the schedule for transmission up to the sink. In this arrangement, data to the sink are periodically transmitted. Depending on each node's computed utility factor, some nodes will assign themselves to the $\mathrm{CH}$, while some will free ride. As packets are transmitted and received at the $\mathrm{CH}$, energy is consumed. The computations of energy consumed during transmission and aggregation of data packets at the $\mathrm{CH}\left(E_{\mathrm{CH}}\right)$ during the formation of the coalition structure and when nodes free ride to the BS/sink $E_{\mathrm{TX}(\mathrm{NC})}$ are defined by equations (8) and (9), respectively. The two equations apply the energy equations of the radio model [26]:

$$
\begin{aligned}
E_{\mathrm{CH}} & =\operatorname{Ln}\left(\left(E_{\text {ele }}+\epsilon_{\mathrm{fs}} d^{2}\right)+E_{\mathrm{RX}(\text { ele })}\right) \\
& =E_{\mathrm{TX}(\mathrm{CH})}+E_{\mathrm{Agg}(\mathrm{CH})}, \\
E_{\mathrm{TX}(\mathrm{NC})} & =\operatorname{Ln}\left(E_{\mathrm{ele}}+\epsilon_{\mathrm{fs}} d^{4}\right),
\end{aligned}
$$

where $L$ represents the data packet being transmitted, $E_{\text {ele }}$ is the electronic energy, $E_{\mathrm{RX}(\mathrm{ele})}$ is the electronic energy consumed as data packets are received, $\epsilon_{\mathrm{fs}}$ denotes amplifier energy of free space, $E_{\text {Agg }}$ is the energy consumed during the data packet aggregation, $n$ is the number of devices (nodes) assigned to the $\mathrm{CH}$, and $d^{2}$ is the distance between the $\mathrm{CH}$ and next hop; it could be $d^{4}$ if $d$ is greater than the threshold $d_{o}$, and this is done based on equation (2).

4.5. Formulation of Contract-Modelled Incentive. The need to sustain cooperative communication calls for special prescription among M2M devices. The invoking of an incentive that is based on the contract-based model is proposed. This section presents the design of the incentive mechanism. The incentive design considers the work proposed in [31, 32]. The parameters that are considered by both the source and the relay devices before venturing into the contract are the basic wage (recharge energy), relay effort, and bonus. In the formulation of the contract model, the interest of both the source and relay needs to be balanced. The following sections highlight the modelling of the source and relay and contract formulation and operations.

4.6. Source Modelling. In this section, we model the optimal achievable energy saving that comes because of the use of the cooperative device in a data packet transmission. Considering the help received from the $i$ th relay device in forwarding data devices, the total achievable energy saving by the source due to cooperation can be expressed as [33]

$$
\pi_{i}=\Phi_{i} E_{i}+\delta
$$

where $\Phi_{i}$ is the profit of each unit of energy and is always greater than zero, $E_{i}$ is the $i$ th relay energy of transmission at the source's receiver, and $\delta$ is the random variable that follows the Gaussian distribution curve, $N\left(\mu, \sigma^{2}\right)$.

Considering the linear sharing scheme, the compensation $W_{i}$ towards the $i$ th relay device is formulated as [34]

$$
W_{i}=\propto_{i}+\beta_{i} E_{i},
$$

where $\propto_{i}$ is the basic recharge energy of the $i$ th zone B's relay device (basic wage) and $\beta_{i}\left(\beta_{i} \in[0,1]\right)$ denotes the bonus (extra recharge energy) based on performance of the relay device as an incentive. The relay devices have different abilities and actions and hence obtain different bonuses. Under cooperation, the source devices strive to achieve maximum utility. The formulation that defines the source's total utility attained with the help of the relay device considers equations $(10)$ and (11). Source utility $\left(U_{s}\right)$ is given as

$$
\begin{aligned}
& U_{\mathrm{s}}=\sum_{i=1}^{n}\left[\pi_{i}-W_{i}\right] \\
& =\sum_{i=1}^{n}\left[\Phi_{i} E_{i}+\delta-\propto_{i}-\beta_{i} E_{i}\right] \text {, } \\
& \text { with means } E\left[U_{\mathrm{s}}\right]=\sum_{i=1}^{n}\left[\Phi_{i} E_{i}-\propto_{i}-\beta_{i} E_{i}\right] \text {, } \\
& \text { and variance } \operatorname{Var}\left[U_{\mathrm{s}}\right]=\sum_{i=1}^{n}[1-\beta]^{2} \sigma^{2} \text {. }
\end{aligned}
$$

The source's expected utility considering a constant absolute risk aversion (CARA) preference that considers equations (13) and (14) is defined as

$$
E\left[U_{s}\right]=E\left[-e^{-\eta s U_{s}}\right]=e^{-\eta s\left[E\left(U_{s}-(1 / 2) \operatorname{Var}\left[U_{s}\right] \eta s\right)\right]} .
$$

4.7. Relay Modelling. The devices in zones B and C are the potential relays to be incentivised in the network. The focus 
is on the input effort and rewards granted to the devices that participate in multihop transmission of data packets to the sink/BS. More energy (effort) used attracts more cost paid by the relay devices. If the channel gain between the source device (SD), and the receiver device (RD) is defined as $h_{\text {(SD,RD) }}$ and the cost of effort used is $C_{i} E_{i}$ and increases with effort, the device communication cost of the $i$ th relay device is assumed to be quadratic and is expressed [32] as

$$
C_{i}\left(E_{i}\right)=\frac{\left(c_{i}\left(E_{i}\right)^{2}\right) / 2}{h_{(\mathrm{SD}, \mathrm{RD})}},
$$

where $c_{i}$ is the cost of energy per unit transmission of the $i$ th relay device. From (16), the hidden information of the $i$ th relay device that depends on the cost of energy of transmission and the channel gains between the source and the receiver can be formulated. The hidden information (battery, memory, and computing power) can be expressed as

$$
\theta_{i}=\frac{c_{i}}{h_{(\mathrm{SD}, \mathrm{RD})}}
$$

When $\theta_{i}$ is low, it means the channel condition of the $i$ th relay device is good, and vice versa.
The presentation of payment by the source device to the $i$ th relay device considers equations (11) and (16) and is defined linearly as [34]

$$
W_{i(\mathrm{RD})}=\propto_{i}+\beta_{i} E_{i}-C_{i} E_{i} .
$$

In the payment offered by the source, bonuses towards the $i$ th relay device are different and follow normal distribution with means that take the following form [32]:

$$
\begin{aligned}
E\left[W_{i(\mathrm{RD})}\right] & =\propto_{i}+\beta_{i} \Phi_{i} E_{i}-C_{i} E_{i}, \\
\operatorname{Var}\left[W_{i(\mathrm{RD})}\right] & =\beta_{i}^{2} \sigma^{2} .
\end{aligned}
$$

If the $i$ th relay device adopts a constant absolute risk aversion (CARA) preference, then the $i$ th device's (RD (receiver device)) utility is expressed as

$$
U\left(W_{i(\mathrm{RD})}\right)=e^{-\eta_{\mathrm{RD}} W_{i(\mathrm{RD})}}
$$

The expected utility for the $i$ th relay device can be expressed as

$$
\begin{aligned}
E\left[U\left(W_{i(\mathrm{RD})}\right)\right] & =E\left[e^{\left.-\eta_{\mathrm{RD}} W_{i(\mathrm{RD})}\right]}\right. \\
& =\frac{-1}{\sqrt{2 \prod \operatorname{Var}\left[W_{i(\mathrm{RD})}\right]}} \int_{-\alpha}^{\alpha} e^{-\left(\left(\left(R_{N}\right)^{2}-2 E\left[W_{i(\mathrm{RD})}\right] W_{i(\mathrm{RD})}+\left(E\left[W_{i(\mathrm{RD})}\right]^{2}\right)+2 \operatorname{Var}\left[R_{N}\right] \eta \mathrm{RD} R_{N}\right) /\left(2 \operatorname{Var}\left[W_{i(\mathrm{RD})}\right]\right)\right) d W_{i(\mathrm{RD})}} \\
& =-e^{-\eta \mathrm{RD}\left[E\left[W_{i(\mathrm{RD})}\right]\right]-(1 / 2) \operatorname{Var}\left[W_{i(\mathrm{RD})}\right] \eta \mathrm{RD}} \\
& =-e^{-\eta \mathrm{RD}\left[\propto_{i}+\beta_{i} \Phi_{i} E_{i}-C_{i} E_{i}-((\eta \mathrm{RD}) / 2) \beta_{i}^{2} \sigma^{2}\right]}
\end{aligned}
$$

4.8. Relay Incentive Mechanism. This section defines the design of contract-modelled incentive between the source device and relay device. The contract formulation considers the monopolistic design technique where the source device proposes a contract item $\left(\alpha_{i}, E_{i}\right)$ that is acceptable by the relay device [32]. In this work, it is assumed that the source knows the ability and action of the existing relay devices (symmetric information). The design will enable the source achieve utility of the relay that is maximum, which when considered under any network information scenario is the upper bound of the source's achievable utility. Under the symmetric information scenario, maximising the expected source utility, equation (15) requires that individual rationality (IR) is satisfied. This can be attained through the derivation starting from

$$
\begin{aligned}
& \max _{\left\{\left\{E_{i} \alpha_{i}\right\} \geq 0\right\}} \quad E\left[U_{s}\right], \\
& \text { s.t. (IR) } \quad \alpha_{i+} \beta_{i} \Phi_{i} E_{i}-C_{i} E_{i}-\frac{\eta \mathrm{RD}}{2} \beta_{i}^{2} \sigma^{2} \geq \widehat{u}, \quad 1 \leq i, \leq n,
\end{aligned}
$$

where $\widehat{u}$ is called the retained utility, the minimum utility level for the source during contract formulation.

Let

$$
\begin{aligned}
\mathrm{fs} & =E\left[U_{\mathrm{s}}\right]-\frac{\operatorname{Var}\left[U_{s}\right] \eta s}{2}, \\
& =\sum_{i=1}^{n}\left[\Phi_{i} E_{i}-W_{i}-\frac{\eta s \sigma^{2}}{2}\left(1-\beta^{\prime}\right)^{2}\right] .
\end{aligned}
$$

The optimisation problem in equation (18) can be written as

$$
\begin{aligned}
& \max _{\left\{\left\{E_{i} \alpha_{i}\right\} \geq 0\right\}} \quad \mathrm{fs}=\sum_{i=1}^{n}\left[\Phi_{i} E_{i}-W_{i}-\frac{\eta s \sigma^{2}}{2}\left(1-\beta^{\prime}\right)^{2}\right], \\
& \text { s.t(IR) } \quad \alpha_{i+} \beta_{i} \Phi_{i} E_{i}-C_{i} E_{i}-\frac{\eta R D}{2} \beta_{i}^{2} \sigma^{2} \geq \widehat{u}, \quad 1 \leq i, \leq n .
\end{aligned}
$$

Considering that there exists an optimal contract item $\left(\alpha_{i}, E_{A(i)}\right)$ that solves the equation $\alpha_{i+} \beta_{i} \Phi_{i} E_{i}-C_{i} E_{i}-$ $(\eta \mathrm{RD} / 2) \beta_{i}^{2} \sigma^{2} \geq \widehat{u}$, the source can obtain maximum utility by 
decreasing $\alpha_{i}$ until the relay device attains the retained utility defined as

$$
\alpha_{i+} \beta_{i} \Phi_{i} E_{i}-C_{i} E_{i}-\frac{\eta \mathrm{RD}}{2} \beta_{i}^{2} \sigma^{2}=\widehat{u}
$$

Considering equation (26), the source's expected utility maximisation problem in equation (25) can be simplified as

$$
\max _{\left\{\left\{E_{i}\right\} \geq 0\right\}}=\sum_{i=1}^{n}\left[\Phi_{i} E_{i}-\widehat{u}-C_{i} E_{i}-\frac{\eta \mathrm{RD}}{2} \beta_{i}^{2} \sigma^{2}-\frac{\eta s \sigma^{2}}{2}\left(1-\beta_{i}\right)^{2}\right] .
$$

The source optimisation problem that had two variables $\left\{E_{i}, \alpha_{i}\right\}$ in (25) is simplified to that with one variable $\left\{E_{i}\right\}$ in (27), which can be computed by interior point techniques [35]. A summary of the operation of contract incentive mechanism can be explained as follows.

The set of source devices, set of relay devices, and set of contract items (energy credits) from the source assumed to be cluster heads are represented as $\mathrm{CH}_{i}, i=\{1,2 \ldots M\}$, $\mathrm{RD}_{S}, S=\{1,2 \ldots P\}$, and $E_{i}$, respectively. The $\mathrm{CH}_{i}$ always strives to maximise $E_{i}$, where minimum energy credits are availed to the RDs; this helps $\mathrm{CH}_{i}$ to minimise its energy expenditure so as to stay in operation for an elevated period. The operation of a contract incentive mechanism starts with the source device broadcasting the contract item $\left(E_{i}\right)$ to the relay devices (RDs). RDs evaluate the $E_{i}$, and the $\mathrm{RD}$ that accepts the broadcasted $E_{i}$ gives feedback. Once $\mathrm{CH}_{i}$ receives a feedback from $\mathrm{RDs}$, it passes the cooperative instructions and required data packets for transmissions. The RDs receive and aggregate the data packets and supplement them with their own and forward to the processing unit/sink. The $\mathrm{CH}_{i}$ rewards $\mathrm{RDs}$ with the agreed $E_{i}$ after successful delivery of the data packets. The operation is illustrated in Algorithm 1.

This section has described the parts of the proposed system model. The next section presents the proposed algorithm CGTCI.

\section{Coalition Game Theoretical Clustering with Incentive (CGTCI) Algorithm}

This section gives a description of the proposed algorithm and analysis of energy consumption during data packet transmissions.

5.1. Description of CGTCI Algorithm. It is assumed that the network area is partitioned into three hierarchical zones: $\mathrm{A}, \mathrm{B}$, and $\mathrm{C}$, with coalition structures forming in zones $\mathrm{A}$ and $\mathrm{B}$, while devices in zone $\mathrm{C}$ communicate directly with the BS. Election of CHs in zones A and B is performed by considering equation (4). A device of high cost function in the zone is elected as a $\mathrm{CH}$. For zone A, the elected $\mathrm{CH}$ selects more devices of high cost function to be sub- $\mathrm{CH}$ to constitute their coalitions. The number of devices selected as sub-CHs should not exceed the number of triangles in the partition. Other devices within the zone not selected as $\mathrm{CH}$ s have an option of joining a coalition or communicating directly to the BS. This is determined by the utility factor of a device that is computed by equations (6) and (7). A high computed utility factor means less energy consumption by the device. When devices get associated with specific $\mathrm{CHs}$ in the zone, coalition structures are generated. The devices forward their data packets to respective $\mathrm{CH}$ in the coalition structures in the zones. The $\mathrm{CHs}$, which are the source device, present the contract items $\left(\alpha_{i}, E_{i}\right)$ to the relay device with expected maximum utility of the source. This is derived through equations (23)-(27). The relay devices evaluate the contract items based on their expected utility derived through equations (19)-(22). The relay devices that accept the contract items receive and aggregate the packets and transmit to the BS/ sink. The flow diagram in Figure 4 illustrates the proposed CGTCI algorithm. The steps of the flow diagram are summarised as follows:

(i) The Sierpinski triangle technique is applied in partitioning the network area into hierarchical zones $\mathrm{A}, \mathrm{B}$, and $\mathrm{C}$. The technique helps in the formation of unequal coalition structures that solve the problem of hot spots.

(ii) Coalition structures are formed after election of CHs.

(iii) Contract-modelled incentive is invoked to stimulate multihop transmissions among the devices in the hierarchical partitioned zones to the BS. A balance is struck between the utility of the source device and the individual rationality (IR) of the potential relay devices at the time of implementing the incentive parameter. An optimal incentive is offered as a contract item to the potential relay devices. The incentive provokes transmissions of data packets through short distances up to the BS and contributes to minimisation of energy consumption in the network.

5.2. Analysis of Energy Consumption Process. The computation of energy consumption applies equations (8) and (9) discussed in Section 4. In this work, for the devices that embrace formation of coalition structures, energy is expended in the following ways: (1) as data packets are transmitted to the $\mathrm{CH}\left(E_{\mathrm{TX}(\mathrm{CH})}\right)$, (2) during data packet aggregation at the $\mathrm{CH}\left(E_{\mathrm{Agg}(\mathrm{CH})}\right)$, and (3) as aggregated data packets are transmitted to the next hop in the next zone/BS $\left(E_{\mathrm{TX}(\mathrm{NH})}\right)$. For the devices that opt for the noncoalition approach, where devices individually transmit data packets to the BS, the energy used for transmission is denoted as $\left(E_{\mathrm{TX}(\mathrm{NC})}\right)$. For the zones far from the BS, the total energy consumed at the time $(t)$ can be computed using the expression

$$
E_{Z}(t)=\left(E_{\mathrm{TX}(\mathrm{CH})}+E_{\mathrm{Agg}(\mathrm{CH})}+E_{\mathrm{TX}(\mathrm{NH})}+E_{\mathrm{TX}(\mathrm{NC})}\right)(t) .
$$

From (28), the computation of energy consumed in zones $\mathrm{A}\left(E_{Z(A)}\right)(t), \mathrm{B}\left(E_{Z(B)}\right)(t)$, and $\mathrm{C}\left(E_{Z(C)}\right)(t)$ can, respectively, be derived from the following expressions: 


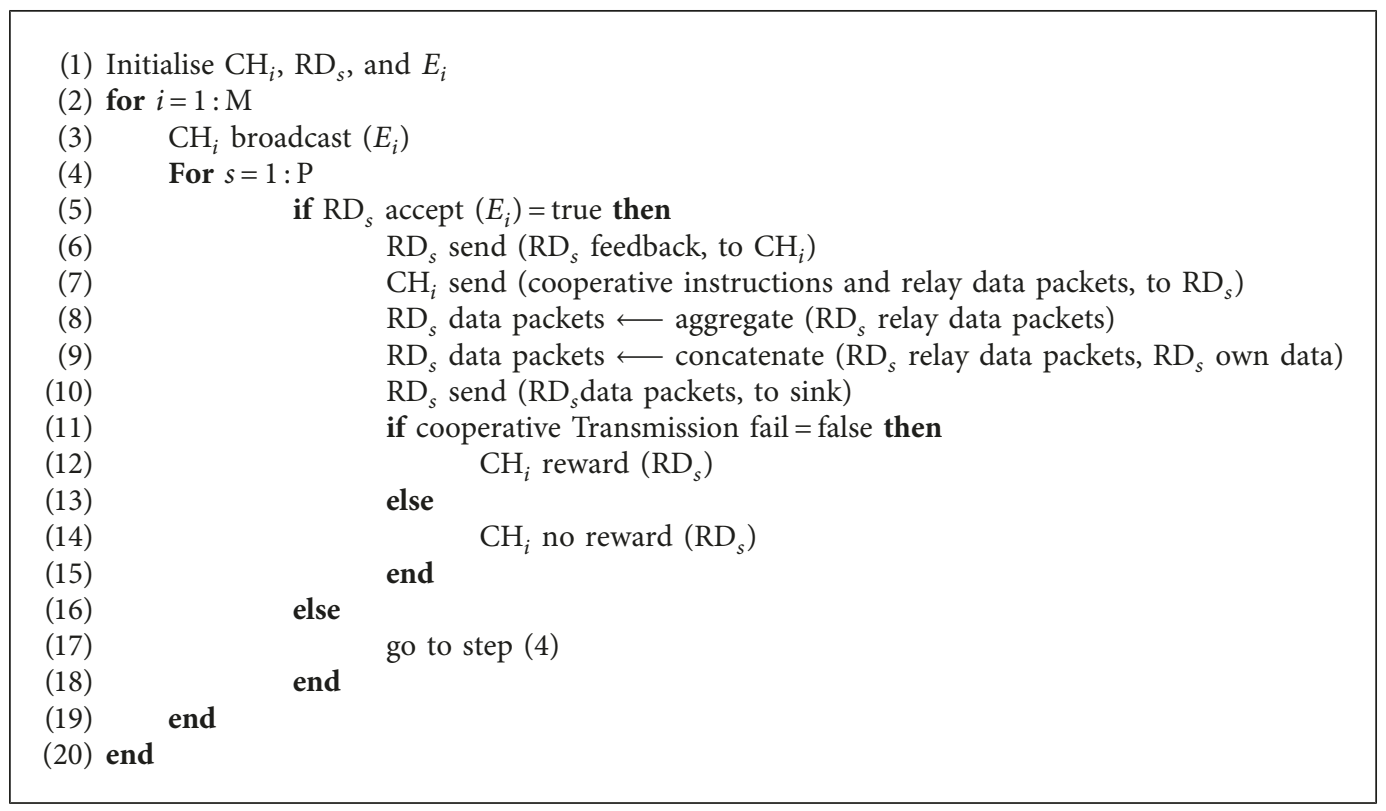

Algorithm 1: Operation of contract incentive mechanism.

$$
\begin{aligned}
E_{Z(A)}(t)= & \left(E_{\mathrm{TX}(\mathrm{CH})[A]}+E_{\mathrm{Agg}(\mathrm{CH})[A]}+E_{\mathrm{TX}(\mathrm{NH})\{B\}}\right. \\
& \left.+E_{\mathrm{TX}(\mathrm{NC})[A]\{\mathrm{BS}\}}\right)(t), \\
E_{Z(B)}(t)= & \left(E_{\mathrm{TX}(\mathrm{CH})[B]}+E_{\mathrm{Agg}(\mathrm{CH})[B]}+E_{\mathrm{Agg}(\mathrm{RD})[A+B]}\right. \\
& \left.+E_{\mathrm{TX}(\mathrm{NH})\{C\}}+E_{\mathrm{TX}(\mathrm{NC})[B]\{\mathrm{BS}\}}\right)(t), \\
E_{Z(C)}(t)= & \left(E_{\mathrm{Agg}(\mathrm{RD})[A+B+C]}+E_{\mathrm{TX}(\mathrm{NH})\{\mathrm{BS}\}}\right. \\
& \left.+E_{\mathrm{TX}(\mathrm{NRD})[C]\{\mathrm{BS}\}}\right)(t),
\end{aligned}
$$

where $E_{\mathrm{TX}(\mathrm{CH})[A]}$ and $E_{\mathrm{TX}(\mathrm{CH})[B]}$ denote energy consumed as data packets are transmitted to the $\mathrm{CH}$ s in zones $\mathrm{A}$ and $\mathrm{B}$, respectively; $E_{\mathrm{Agg}(\mathrm{CH})[A]}, E_{\mathrm{Agg}(\mathrm{CH})[B]}$, and $E_{\mathrm{Agg}(\mathrm{RD})[A+B+C]}$ denote energy consumed during data aggregation at $\mathrm{CH}$ in zones $\mathrm{A}$ and $\mathrm{B}$ and at the relay device in zone $\mathrm{C}$, respectively; $E_{\mathrm{TX}(\mathrm{NH})\{B\}}, E_{\mathrm{TX}(\mathrm{NH})\{\mathrm{C}\}}$, and $E_{\mathrm{TX}(\mathrm{NH})\{\mathrm{BS}\}}$ denote energy consumed as data packets are transmitted to the next hop B, C, and $\mathrm{BS}$, respectively; and $E_{\mathrm{TX}(\mathrm{NC})[A]\{\mathrm{BS}\}}, E_{\mathrm{TX}(\mathrm{NC})[B]\{\mathrm{BS}\}}$, and $E_{\mathrm{TX}(\mathrm{NRD})[\mathrm{C}]\{\mathrm{BS}\}}$ represent energy consumed from devices in zones A and B that are not organised in coalition structures as they transmit data packets to the BS and devices in zone $\mathrm{C}$ that are not selected as relay devices as they transmit data packets to the BS.

Total energy consumed in one round of data packet transmission to the BS at the time $(t)$ is obtained as a summation of energies defined in equations (29)-(31):

$$
E_{T}(t)=\left(E_{Z(A)}+E_{Z(B)}+E_{Z(C)}\right)(t) .
$$

This section has highlighted the proposed CGTCI algorithm and how energy consumed in one round of data packet transmission is computed. In the next section, performance evaluation of the algorithm is presented.

\section{Performance Evaluation}

This section presents the simulation parameters, scenarios, and results.

6.1. Simulation Parameters. The simulation of the CGTCI is carried out in the MATLAB software environment. Three parameters are considered: total energy trend in the network, energy trend for the farthest located device from the BS, and number of surviving devices in relation to the number of rounds of packet transmissions. 200 devices are considered, and the BS is located at $(10,000,10,000)$ metres. It is assumed that packets are all delivered successfully to their respective destinations, and interference in the network is assumed to be fixed and equal for all the nodes. The CGTCI algorithm is evaluated for energy efficiency, and it is compared with CGTC [15], CG-DC that is developed from the improved low-energy adaptive clustering hierarchy (LEACH) [16], and noncoalition game (NCG) algorithms. The total initial energy in the system is 2300 joules. Table 1 presents other parameters utilised in the simulation that are comparable to those presented in [15].

6.2. Simulation Scenarios. The data transmission scenarios for NCG, CGTC/CGTCI, and CG-DC algorithms are presented in the following sections.

6.3. NCG Simulation Scenario. Figure 5 illustrates the NCG simulation scenario where each device transmits its own data packets to the BS. 


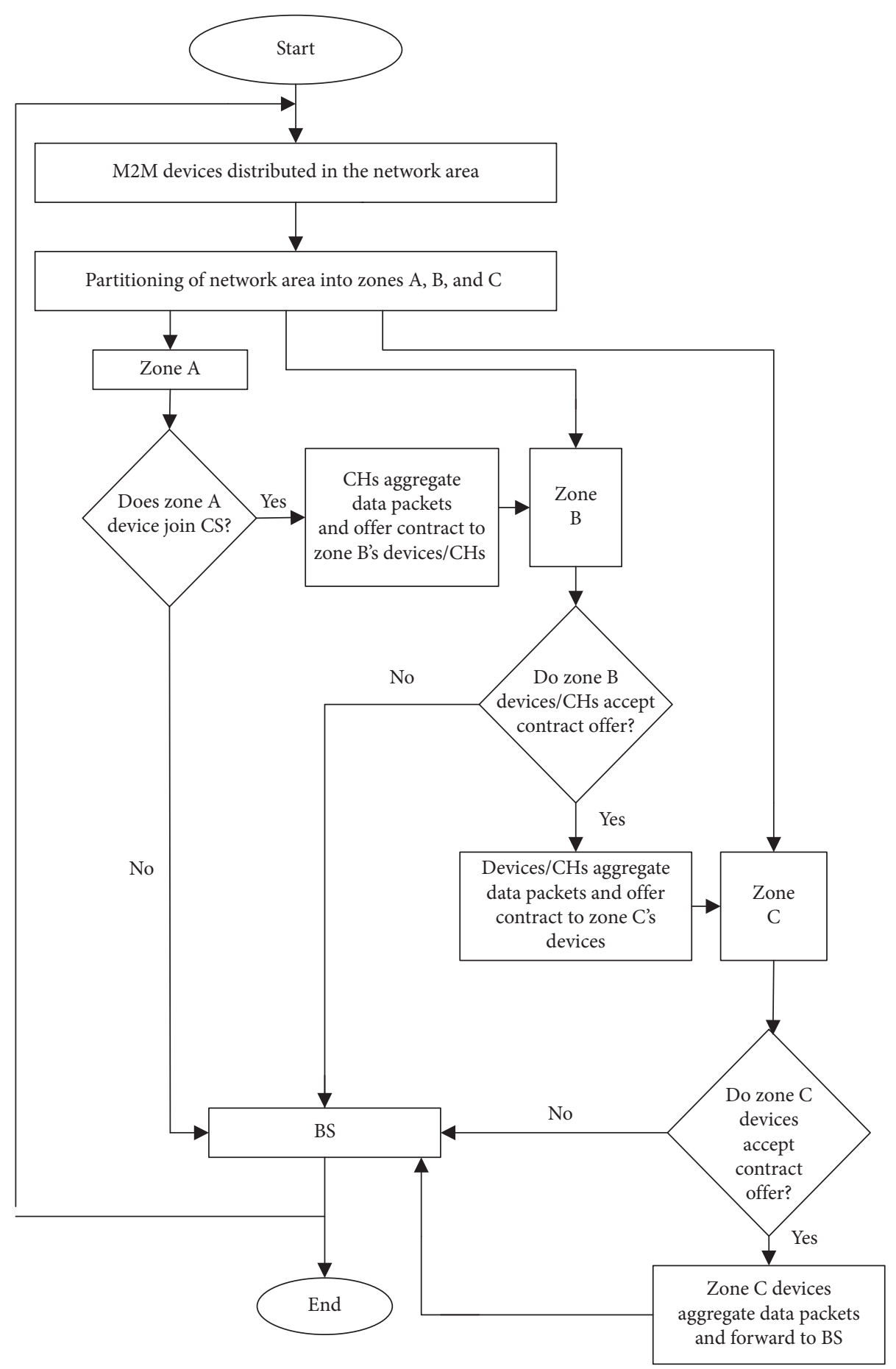

FIgURE 4: Flow chart of the CGTCI algorithm.

6.4. CGTC/CGTCI Simulation Scenario. CGTC and CGTCI have a similar structural arrangement that is shown in Figure 6. In the outer and middle zones, the main $\mathrm{CH}$ is elected, which then selects other sub-CHs whose cost functions are above the average threshold. The inner zone devices are not organised in coalition structures. The devices in the outer and middle zones then apply the utility equations (6) and (7) to select specific CHs in the zone where their utility factor score is the highest. The CGTCI algorithm has data packet transmission from three regions: the inner, middle, and outer zones. (i) For the inner zone, the devices communicate directly with the BS. (ii) For the middle zone, after the CHs aggregate data packets of their members, they invoke a contract-modelled incentive. The $\mathrm{CHs}$ broadcast their contract items to the inner zone devices, and the contract items are evaluated. Those that accept the contract items receive and aggregate the data packets, which they forward to the BS. (iii) For the outer zone, first there is an 
TABLe 1: Simulation parameters.

\begin{tabular}{lcc}
\hline Parameter & Value & Description \\
\hline$N$ & 200 & Number of nodes \\
BS (location) & $(10,000,10,000)$ & Location of BS in metres \\
BS (cov.) & $14,000 \mathrm{~m}$ & Radius of BS coverage \\
$L$ & $0-1000$ & Data payload in bits (varying) \\
$\varepsilon_{\mathrm{fs}}$ & $1 * 10^{-12} \mathrm{~J} / \mathrm{bit} / \mathrm{m}^{2}$ & Amplification energy of free space \\
$\varepsilon_{\mathrm{tr}}$ & $1.3 * 10^{-15} \mathrm{~J} / \mathrm{bit} / \mathrm{m}^{4}$ & Amplification energy of multipath \\
$\mathrm{En} / \mathrm{b}$ & $50 * 10^{-9} \mathrm{~J}$ & Energy/bit \\
$E_{\mathrm{agg} .}$ & $5 * 10^{-9} \mathrm{~J}$ & Data aggregation energy \\
$d_{o}$ & $87 \mathrm{~m}$ & Threshold value $\left(\sqrt{\left(\epsilon_{\mathrm{fs}} / \epsilon_{\mathrm{mp}}\right)}\right)$ \\
$E_{i}$ & $0-20$ joules & Device energy \\
$E_{D}$ & $0-20$ joules & ith relay energy from the energy) \\
\hline
\end{tabular}

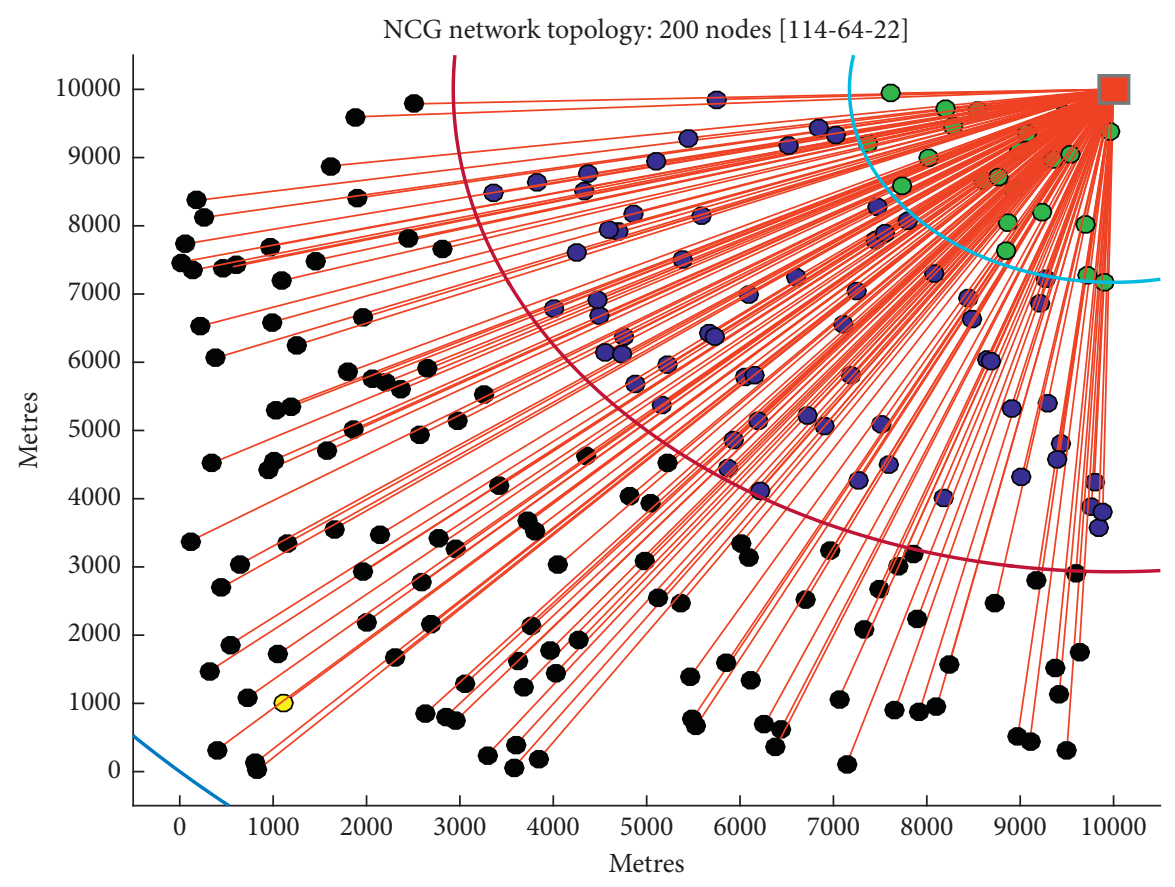

FIGURE 5: NCG simulation scenario.

internal multihop transmission of data packets, where the specific sub-CHs after receiving and aggregating data packets of their members establish a route through other sub-CHs in the zone until they reach the main $\mathrm{CH}$. The main $\mathrm{CHs}$ aggregate the data packets and broadcast the contract items to the devices in the middle zone. The device in the middle zone that accepts the contract items repeats the procedures in (ii). For the CGTC algorithm, the operation is like the procedures of CGTCI but lacks the engagement of the incentive.

6.5. CG-DC Simulation Scenario. Figure 7 illustrates the simulation scenario of coalition game-direct communication (CG-DC). In this arrangement, elected $\mathrm{CH}$ receive data packets from members of the coalition structure and forward them directly to the BS, with no multihop communication.
6.6. Simulation Results. This section describes sample simulated results, and energy efficiency of the algorithms is defined based on the following:

(i) The trend of computed average energy remaining in the network as the number of rounds of packet transmissions increases

(ii) The trend of computed average energy in the network for the devices located in the farthest zone from the BS as the number of rounds of packet transmissions increases

(iii) The trend of computed average energy in the network for the devices located in the zone closest to the BS

(iv) The number of nodes remaining in the network as the number of rounds of data packet transmissions increases 


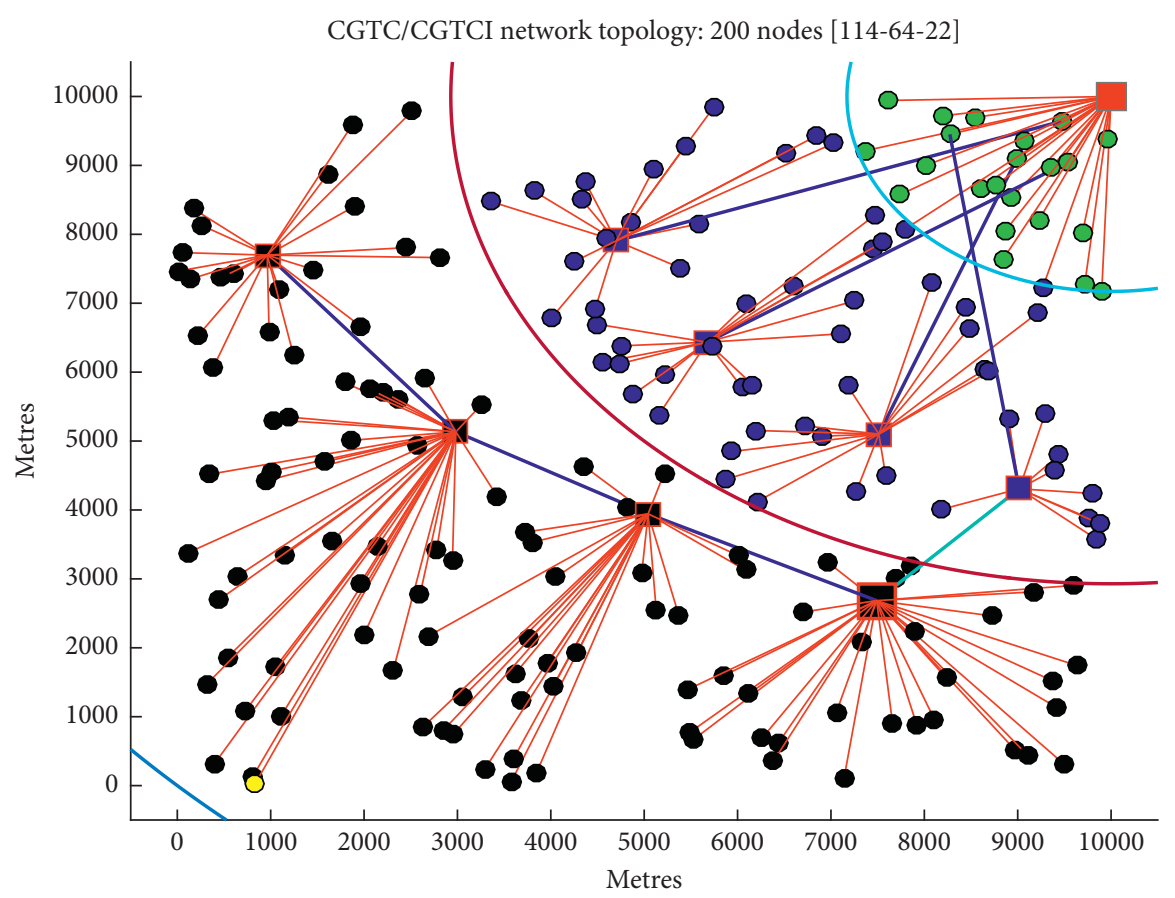

Figure 6: CGTC/CGTCI simulation scenario.

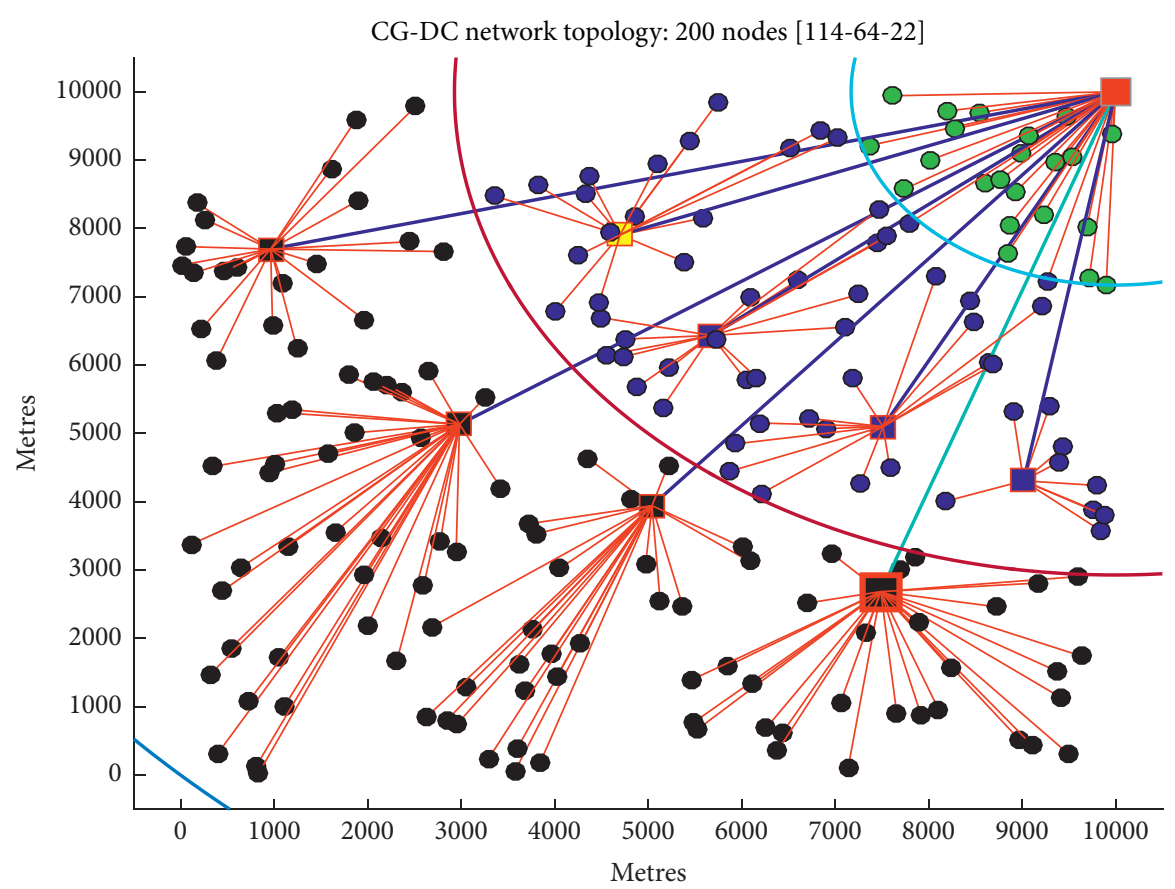

FIGURE 7: CG-DC simulation scenario.

6.6.1. Trend of Total Energy Remaining in the Network. Figure 8 shows that as the number of rounds of packet transmissions increases, there is a steep decrease in energy in NCG when compared with CGTC, CG-DC, and CGTCI. The decrease in energy displayed by the CGTCI algorithm is less when compared with CGTC, CG-DC, and NCG. At the 5000th round of packet transmission, when the NCG algorithm has approximately $0 \%$ remaining energy in the network, CGTCI has approximately 20\% remaining energy, while CGTC and CG-DC display approximately $10 \%$ energy remaining in the network. Figure 9 displays the percentages of the remaining energy at the 5000th round of packet transmission with NCG as a reference. The observation is attributed to the engagement of a contract-modelled incentive which stimulates the devices in the respective zones to accept a multihop mode of transmissions. This enables 


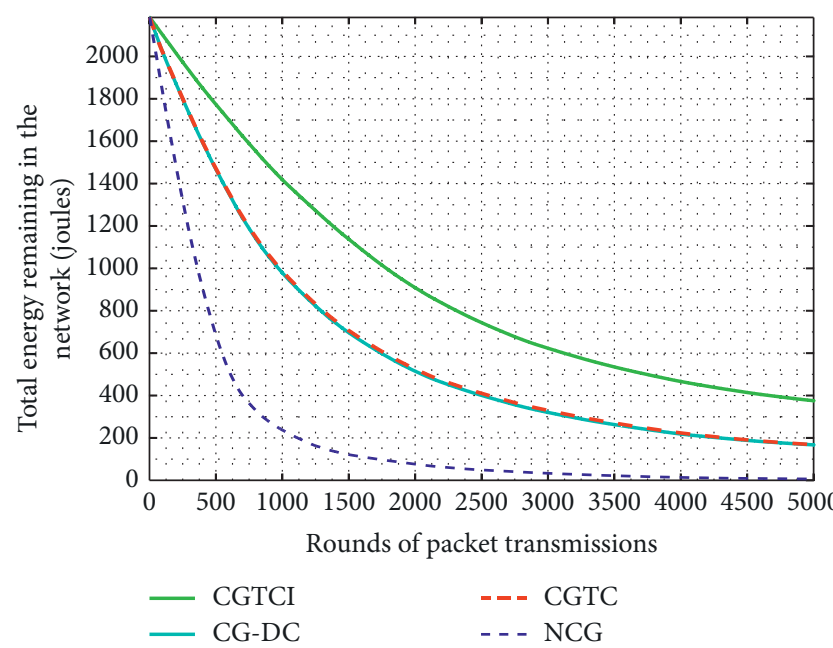

Figure 8: The trend of total energy remaining in the network.

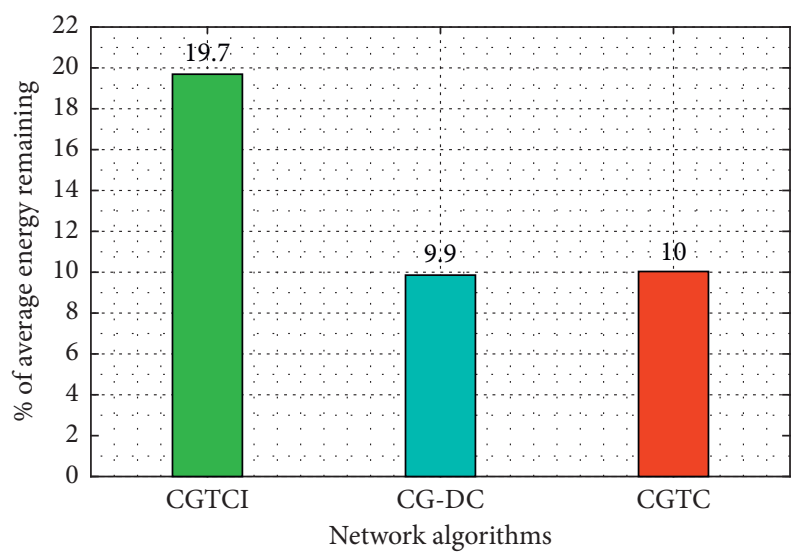

FIGURE 9: \% of average remaining energy.

data packets to be moved from the outer zones to the BS through short-distance transmissions, consuming less energy. The network design that considers the CGTC algorithm subjects its inner zone devices (zone C) to heavy energy drain as it receives and forwards data packets to the sink. The inner zone devices are not compensated for the heavy work they do and therefore become eliminated. In the CG-DC algorithm, outer zone devices (zones A and B) drain a lot of energy as they transmit data packets through long distances to the BS, and there is no multihop transmission. NCG displays a steep decrease in the remaining energy than all the given algorithms. This is attributed to the high energy consumption in the far located devices as they transmit data to the BS. Interferences and collisions experienced during the time of transmission and accessing the BS also contribute to high energy consumption in the NCG algorithm.

6.6.2. Energy Trend for the Devices Located Farthest from the $B S$. Figure 10 compares the trend of the remaining energy for a cluster of devices located in the farthest zone from the BS. The trend of the remaining energy in the devices is analysed when each of the following network designs: CGTCI, CGTC, CG-DC, and NCG, is considered during data packet transmissions in a given period of simulation. With the initial energy of the device at 16.5 joules, the average remaining energy for devices sampled at 50th, 100th, 150 th, and 200th rounds of packet transmissions is $95 \%$, 93\%, $92 \%$, and $88.5 \%$ for CGTCI, CGTC, CG-DC, and NCG, respectively. This indicates that the CGTCI algorithm is $2 \%$, $3 \%$, and $6.5 \%$ better than CGTC, CG-DC, and NCG, respectively.

The formation of the coalition structure in CGTCI and the invoking of a contract-modelled incentive scheme pave way for the short-distance transmissions on the network up to the sink/BS. The short-distance transmission reduces energy consumption, which makes the CGTCI algorithm suitable for devices located farthest from a BS in M2M communications. Multihop transmissions are observed in CGTC; however, lack of incentive does not guarantee that the next hop is necessarily of the shortest distance. This makes the remaining energy level for the device considered under CGTC design to be lower than that when the device is considered under CGTCI design. For the CG-DC algorithm, the device's remaining energy level is slightly lower than that in CGTC but higher than that in the NCG algorithm. The farthest located device in the NCG algorithm experiences a steady decline in the energy level. The data are transmitted through a long distance and result in high energy consumption.

6.6.3. Energy Trend for the Devices Located Closest to the BS (Inner Zone). The trend of the remaining energy in the devices located close to the BS is as shown in Figure 11. The performance of the CGTCI algorithm is better than that of CGTC, CG-DC, and NCG algorithms. At the end of the simulation period, the CGTCI algorithm displays a higher level of remaining energy. This is due to the contractmodelled incentive operation invoked in the CGTCI algorithm that enables compensation of energy used by the devices selected as relays. For the CG-DC algorithm, the energy trend is gradual, but it is at a higher level than that in NCG at the end of the simulation period. This is due to the formation of coalition structures in CG-DC that are supervised by cluster heads. This helps to minimise the number that can access the sink/BS in the CG-DC algorithm when compared to the NCG algorithm and therefore assists to reduce energy loss due to collision. For the NCG algorithm, the high number of devices that try to access the sink/BS results in collision and hence energy loss. Therefore, apart from losing energy due to the transmission of data packets to the BS, energy is also lost during resending of data packets lost through collision. This makes the remaining energy level of the NCG algorithm to be lower than that of the CG-DC algorithm but higher than that of the CGTC algorithm.

For CGTC, the lack of incentive scheme means that the devices selected as relay devices do not compensate for the energy used in relaying. Therefore, the selected devices closer to the BS become exhausted much earlier. The heavy drain of energy from the devices that act as relays contributes to the 


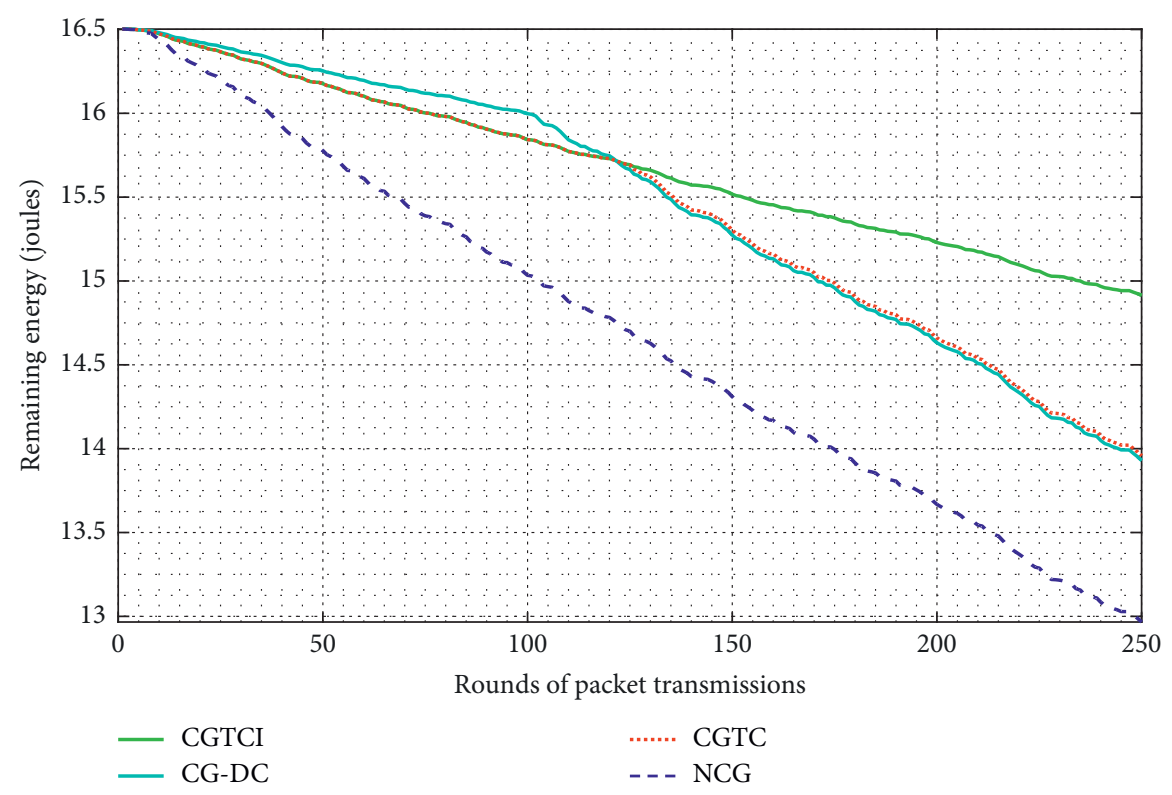

FIgURE 10: Trend of the remaining energy for the devices located farthest from the BS.

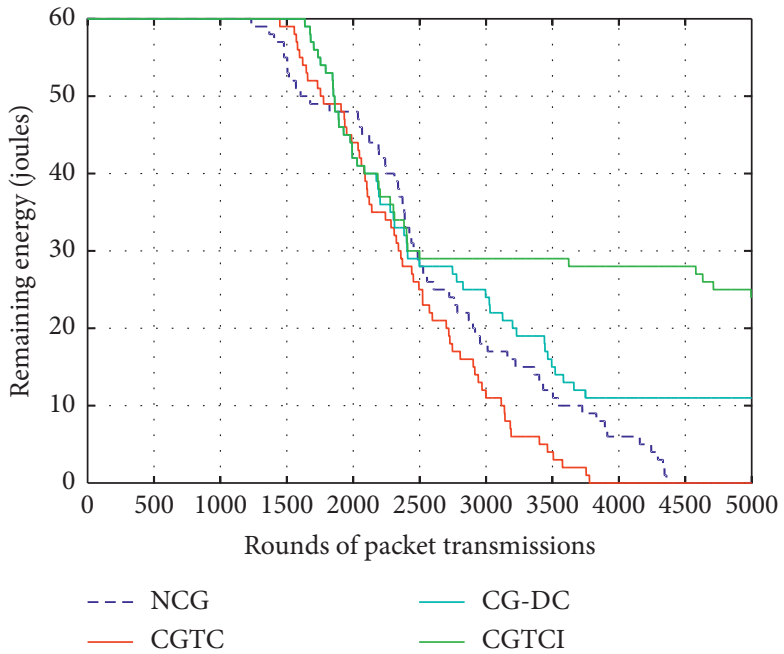

FIgURE 11: Energy trend for the devices located closest to the BS.

trend of the remaining energy for the devices closer to the BS in the CGTC algorithm to be lower than that in all the algorithms by the end of the simulation period. From the observation above, the CGCTI algorithm presents energyefficient results for the devices located closer to the BS than its closely related algorithms-CGTC, CG-DC, and NCG.

6.6.4. Number of Surviving Devices in the Network. The number of surviving devices (nodes) decreases in all the algorithms as the number of rounds of transmissions increases. However, CGTCI has a higher number of surviving nodes when compared with CGTC, CG-DC, and NCG at any given round of packet transmission. Figure 12 displays the number of surviving devices against rounds of packet transmissions. The incentive scheme adopted in the CGTCI algorithm that is absent in CGTC, CG-DC, and NCG enables it to have a balanced energy consumption. As a result, the death rate of devices in the network that adopts the CGTCI algorithm in the design is reduced. The network design that applies the NCG technique posts poor results in terms of the number of surviving devices against the number of rounds of packet transmissions when compared with CGTCI. At the 5000th round of packet transmission, CGTCI has 45, CGTC and CG-DC have 20, and NCG has 0 numbers of surviving devices out of the initial 200. This translates to $22.5 \%, 12.5 \%$, and $0 \%$ number of surviving devices for CGTCI, CGTC and CG-DC, and NCG, respectively. The number of surviving devices reduces at a fast rate in the network design that applies the NCG design. This is due to high energy consumption by the devices as they transmit data packets through a long distance to the BS. CGTC and CG-DC 


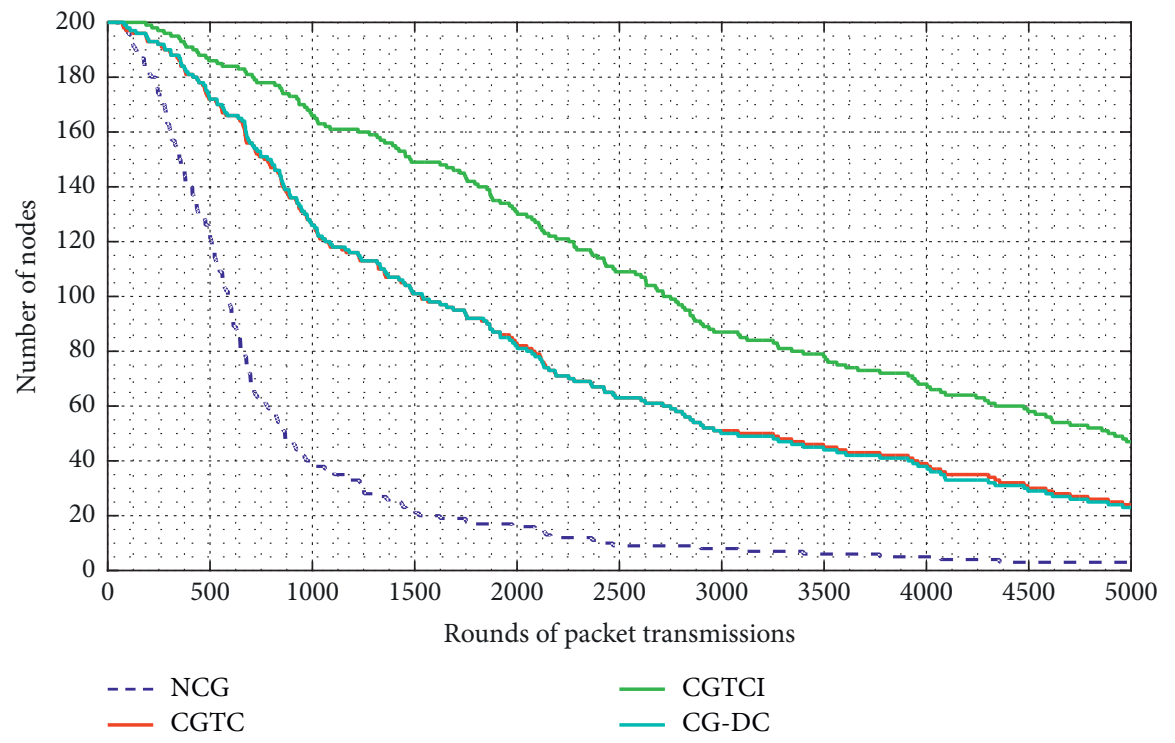

FIgURE 12: Trend of the number of surviving devices in the network.

display almost similar performance. In the CGTC algorithm, the creation of hot spot areas in zone $\mathrm{C}$ contributes to high elimination of devices. In the CG-DC algorithm, the lack of multihop transmission means that devices located far from the BS consume more energy during data transmissions and therefore die early.

\section{Conclusions and Future Work}

A coalition game theoretical clustering with incentive (CGTCI) was proposed for M2M communications. The main strategy was to prolong the network lifetime by controlling the energy consumption as devices transmit data packets to the BS. The network was partitioned into three zones with the help of the Sierpinski triangle. Coalition structures were formed in zones $\mathrm{A}$ and $\mathrm{B}$, while in zone $\mathrm{C}$, the devices free ride to the BS. A contract-based model incentive scheme to stimulate cooperation is invoked. The simulation results indicate that the CGTCI algorithm is better since it has, on average, $10 \%$ more remaining energy and number of surviving devices than its closely related algorithm, CGTC, at the end of simulations. From the displayed results, the CGTCI algorithm is suitable for an energy-efficient M2M communication. Being an energy-efficient algorithm, CGTCI can help maintain the required quality of service for an elevated period than CGTC.

As a further improvement of this work, the $\mathrm{CH}$ selection approach will be examined to optimize both time and energy that arises due to signalling. There is also a need to investigate the reliability and latency of the algorithm to ascertain its performance in packet delivery to its required destinations.

\section{Data Availability}

The data used to support the findings of this study are included within the article.

\section{Conflicts of Interest}

Regarding the publication of this research article, the authors declare that they have no conflicts of interest.

\section{Acknowledgments}

The authors would like to thank the French South African Institute of Technology (F'SATI) and the Tshwane University of Technology for their material and financial support during the production of this work.

\section{References}

[1] H. Zhou, H. Wang, X. Chen, X. Li, and S. Xu, "Data offloading techniques through vehicular ad hoc networks: a survey," IEEE Access, vol. 6, pp. 65250-65259, 2018.

[2] C.-M. Huang, Y.-F. Chen, S. Xu, and H. Zhou, "The vehicular social network (VSN)-Based sharing of downloaded geo data using the credit-based clustering scheme," IEEE Access, vol. 6, pp. 58254-58271, 2018.

[3] J. W. Raymond, T. O. Olwal, and A. M. Kurien, "Cooperative communications in machine to machine (M2M): solutions, challenges and future work," IEEE Access, vol. 6, pp. 97509766, 2018.

[4] T. O. Olwal, K. Djouani, and A. M. Kurien, "A survey of resource management toward 5G radio access networks," IEEE Communications Surveys \& Tutorials, vol. 18, no. 3, pp. 1656-1686, 2016.

[5] D. Lin and Q. Wang, "A game theory based energy efficient clustering routing protocol for WSNs," Wireless Networks, vol. 23, no. 4, pp. 1101-1111, 2017.

[6] R. B. Myerson, Game Theory: Analysis of Conflict, Harvard University Press, Cambridge, MA, USA, 1997.

[7] P. Raja and P. Dananjayan, "Game theory-based efficient energy consumption routing protocol to enhance the lifetime of WSN," International Journal of Information and Communication Technology, vol. 8, no. 4, pp. 357-370, 2016.

[8] F. Li, G. Chang, L. Yao, and F. Gao, "Cooperative gamebased routing approach for wireless sensor network," International 
Journal of Computer Applications in Technology, vol. 44, no. 2, pp. 101-108, 2012.

[9] W. Saad, Z. Han, M. Debbah, A. Hjorungnes, and T. Basar, "Coalitional game theory for communication networks: a tutorial," IEEE Signal Processing Magazine, vol. 26, no. 5, pp. 77-97, 2009.

[10] G. Bacci, S. Lasaulce, W. Saad, and L. Sanguinetti, "Game theory for networks: a tutorial on game-theoretic tools for emerging signal processing applications," IEEE Signal Processing Magazine, vol. 33, no. 1, pp. 94-119, 2016.

[11] T. AlSkaif, M. G. Zapata, and B. Bellalta, "Game theory for energy efficiency in wireless sensor networks: latest trends," Journal of Network and Computer Applications, vol. 54, pp. 33-61, 2015.

[12] A. C. Voulkidis, M. P. Anastasopoulos, and P. G. Cottis, "Energy efficiency in wireless sensor networks: a gametheoretic approach based on coalition formation," $A C M$ Transactions on Sensor Networks (TOSN), vol. 9, no. 4, pp. 1-27, 2013.

[13] D. B. Johnson and D. A. Maltz, "Dynamic source routing in ad hoc wireless networks," in Mobile Computing, pp. 153-181, Springer, Berlin, Germany, 1996.

[14] P. Michiardi and R. Molva, "Simulation-based analysis of security exposures in mobile ad hoc networks," in Proceedings of the European Wireless Conference, pp. 15-17, Florence, Italy, September 2002.

[15] M. Afsar, "Energy-efficient coalition formation in sensor networks: a game-theoretic approach," https://arxiv.org/abs/ 1512.08019 .

[16] J. Xu, N. Jin, T. Peng, and Q. Zhou, "Improvement of LEACH protocol for WSN," in Proceedings of the 2012 9th International Conference on Fuzzy Systems and Knowledge Discovery, FSKD 2012, pp. 2174-2177, Chongqing, China, May 2012.

[17] L. Yang, Y. Z. Lu, Y. C. Zhong, X. G. Wu, and S. J. Xing, “A hybrid, game theory based, and distributed clustering protocol for wireless sensor networks," Wireless Networks, vol. 22, no. 3, pp. 1007-1021, 2016.

[18] K. Yue, J. Zhang, J. Li, T. Wu, and W. Liu, "A theoretic approach for prolonging lifetime of wireless sensor networks based on the coalition game model," International Journal of Distributed Sensor Networks, vol. 10, no. 6, article 328710, 2014.

[19] T. Wu, K. Yue, W. Liu, and J. Xu, “An energy-efficient data transfer model of wireless sensor networks based on the coalitional game theory," in Proceedings of the Eighth International Conference on Fuzzy Systems and Knowledge Discovery (FSKD), pp. 1354-1358, Shanghai, China, July 2011.

[20] H. Jing and H. Aida, "Cooperative clustering algorithms for wireless sensor networks," in Smart Wireless Sensor Networks, InTech, London, UK, 2010.

[21] X.-N. Miao and G. Xu, "Cooperative differential game model based on trade-off between energy and delay for wireless sensor networks," Annals of Operations Research, vol. 206, no. 1, pp. 297-310, 2013.

[22] D. W. K. Yeung and L. A. Petrosjan, Cooperative Stochastic Differential Games, Springer Science \& Business Media, Berlin, Germany, 2006.

[23] L. Tan, S. Zhang, and J. Qi, "Cooperative cluster head selection based on cost sharing game for energy-efficient wireless sensor networks," Journal of Computational Information Systems, vol. 8, no. 9, pp. 3623-3633, 2012.
[24] M. Mishra, CR. Panigrahi, and J. L. Sarkar, "GECSA: a game theory based energy efficient cluster-head selection approach in wireless sensor networks," in Proceedings of the 2015 International Conference on Man and Machine Interfacing (MAMI), pp. 1-5, Bhubaneswar, India, December 2015.

[25] E. Romero, J. Blessa, A. Araujo, and O. Nieto-Taldriz, "A game theory based strategy for reducing energy consumption in cognitive WSN," International Journal of Distributed Sensor Networks, vol. 10, no. 1, article 965495, 2014.

[26] W. R. Heinzelman, A. Chandrakasan, and H. Balakrishnan, "Energy-efficient communication protocol for wireless micro sensor networks," in Proceedings of the 33rd Annual Hawaii International Conference on System Sciences, p. 10, Maui, HI, USA, January 2000.

[27] L. Liang, L. Xu, B. Cao, and Y. Jia, "A cluster-based congestion-mitigating access scheme for massive M2M communications in internet of things," IEEE Internet of Things Journal, vol. 5, no. 3, pp. 2200-2211, 2018.

[28] A. B. F. Guiloufi, N. Nasri, and A. Kachouri, "An energyefficient unequal clustering algorithm using Sierpinski Triangle for WSNs," Wireless Personal Communications, vol. 88, no. 3, pp. 449-465, 2016.

[29] W. B. Heinzelman, A. P. Chandrakasan, and H. Balakrishnan, "An application-specific protocol architecture for wireless micro sensor networks," IEEE Transactions on Wireless Communications, vol. 1, no. 4, pp. 660-670, 2002.

[30] F. Kazemeyni, E. B. Johnsen, O. Owe, and I. Balasingham, "Group selection by nodes in wireless sensor networks using coalitional game theory," in Proceedings of the 2011 16th IEEE International Conference on Engineering of Complex Computer Systems, pp. 253-262, Las Vegas, NV, USA, April 2011.

[31] N. Zhao, M. Wu, W. Xiong, and C. Liu, "Cooperative communication in cognitive radio networks under asymmetric information: a contract-theory based approach," International Journal of Distributed Sensor Networks, vol. 2015, pp. 1-11, 2015.

[32] N. Zhao, Y. Chen, R. Liu, M. Wu, and W. Xiong, "Monitoring strategy for relay incentive mechanism in cooperative communication networks," Computers \& Electrical Engineering, vol. 60, pp. 14-29, 2017.

[33] J. N. Laneman and G. W. Wornell, "Distributed space-timecoded protocols for exploiting cooperative diversity in wireless networks," IEEE Transactions on Information Theory, vol. 49, no. 10, pp. 2415-2425, 2003.

[34] R. Gibbons, "Incentives between firms (and within)," Management Science, vol. 51, no. 1, pp. 2-17, 2005.

[35] S. Boyd and L. Vandenberghe, Convex Optimization, Cambridge University Press, Cambridge, UK, 2004. 


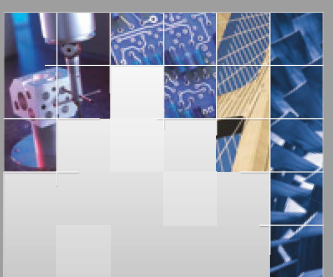

\section{Enfincering}
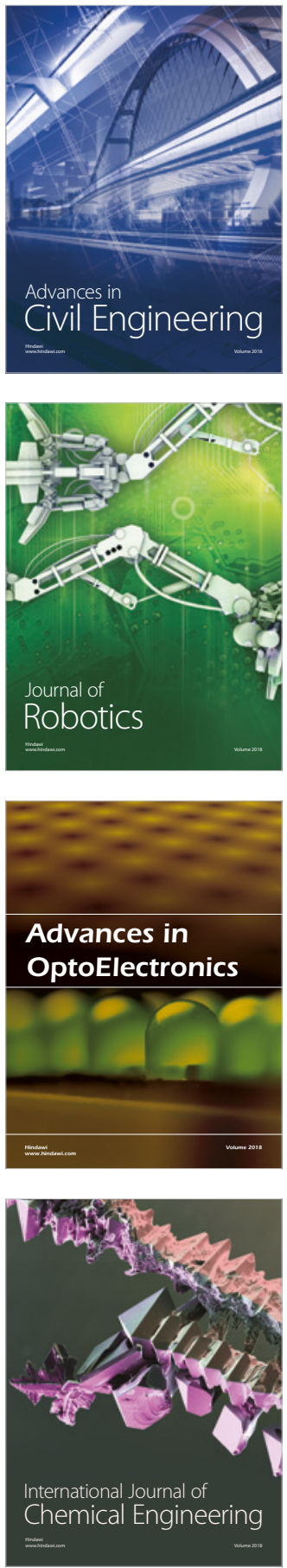

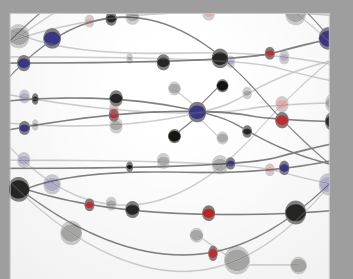

\section{Rotating \\ Machinery}

The Scientific World Journal

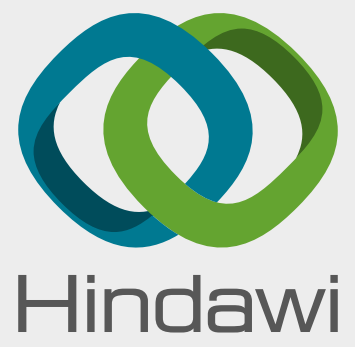

Submit your manuscripts at

www.hindawi.com
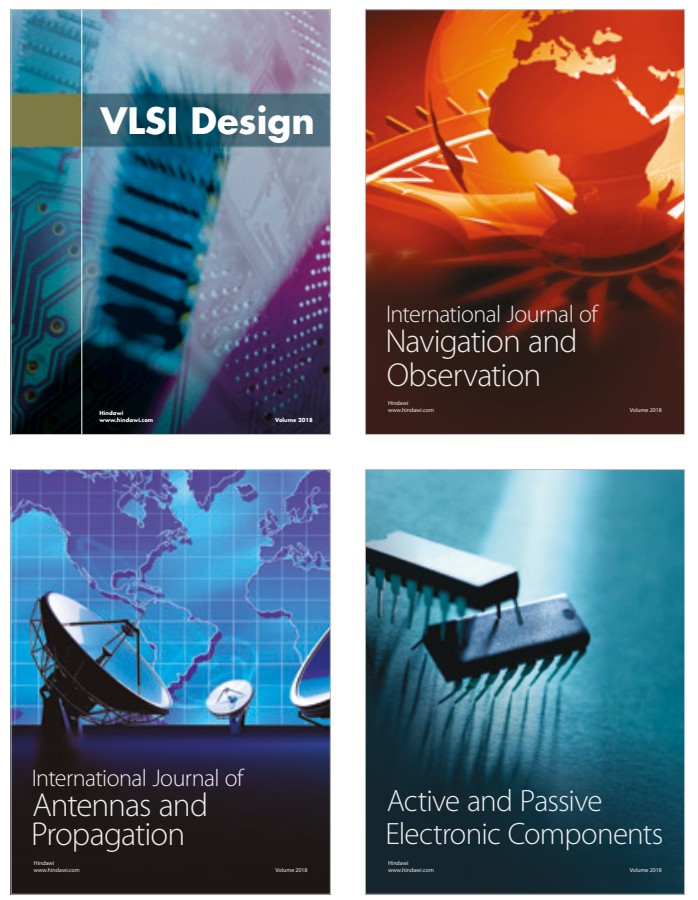
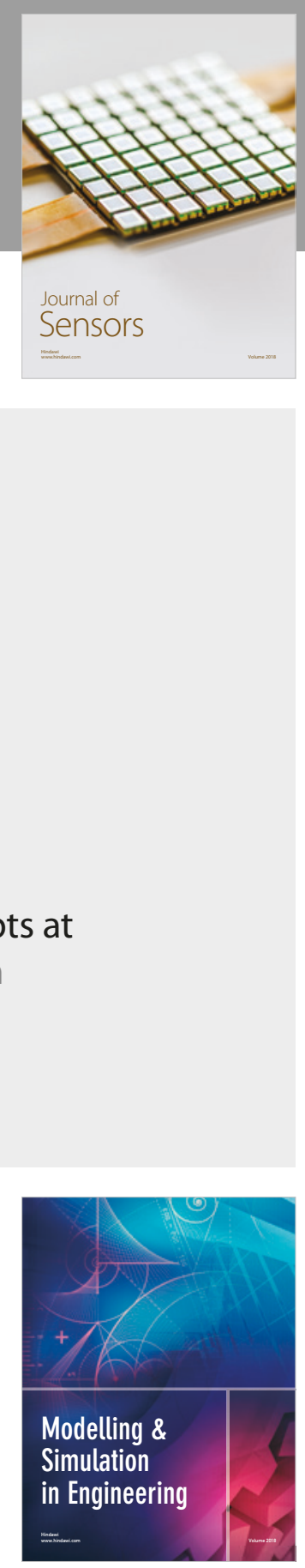

\section{Advances \\ Multimedia}
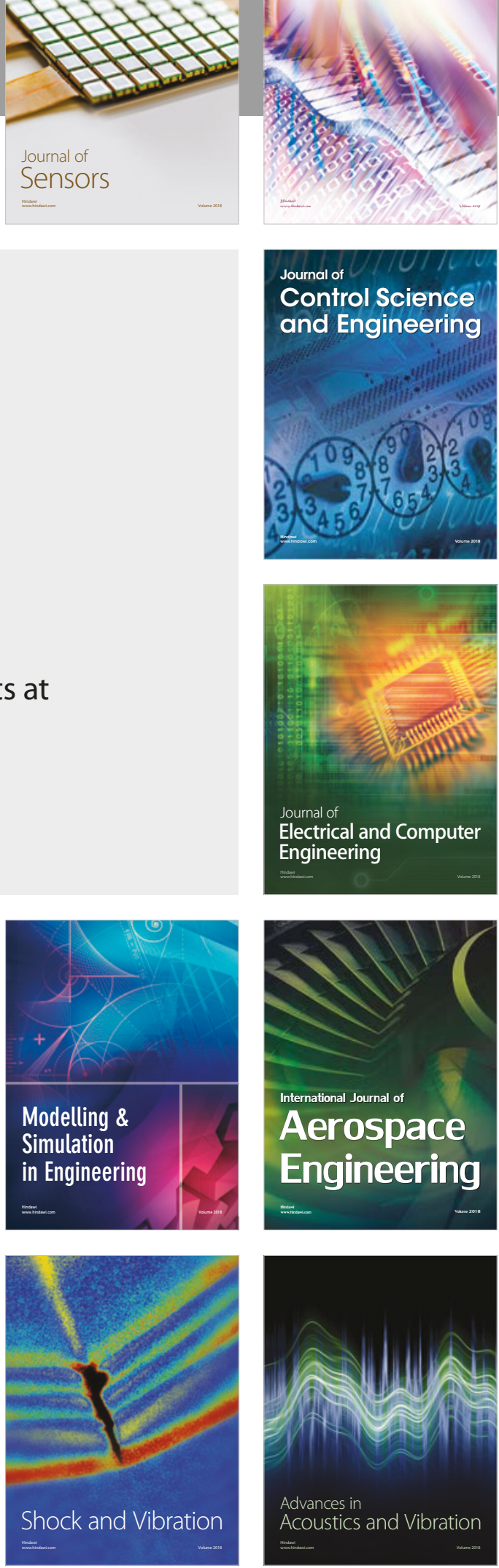